PROCEEDINGS OF THE

AMERICAN MATHEMATICAL SOCIETY

Volume 127, Number 1, January 1999, Pages 89-94

S 0002-9939(99)04338-5

\title{
PERIODIC SOLUTIONS FOR NONCONVEX DIFFERENTIAL INCLUSIONS
}

\author{
SHOUCHUAN HU, DIMITRIOS A. KANDILAKIS, AND NIKOLAOS S. PAPAGEORGIOU
}

(Communicated by Hal L. Smith)

\begin{abstract}
In this paper we prove the existence of periodic solutions for differential inclusions with nonconvex-valued orientor field. Our proof is based on degree theoretic arguments.
\end{abstract}

\section{INTRODUCTION}

In a recent paper Hu-Papageorgiou [6] proved the existence of a periodic solution for a nonconvex differential inclusion in $R^{N}$. To the best of our knowledge, this was the first existence result for nonconvex periodic problems. All earlier works had assumed that the orientor field (multivalued vector field) is convex valued. We refer to the works of Haddad-Lasry [4], Aubin-Cellina [1], Macki-Nistri-Zecca [7] and Plaskacz [11]. The approach of Hu-Papageorgiou [6] was based on directionally continuous selectors for the orientor field and on a Nagumo type tangential condition. In this paper our approach is different and is based on degree theoretic arguments. So our hypotheses on the orientor field are different, in some respects weaker, than the ones used by Hu-Papageorgiou [6] (compare the growth hypothesis $\mathrm{H}(\mathrm{F})$ (iii) here and in [6]) and also uses a function like the guiding function of Mawhin [8], which gives us a priori bounds and thus makes possible the use of degree theory.

\section{Preliminaries}

In what follows by $P_{k}\left(R^{N}\right)$ we will denote the collection of all nonempty and compact subsets of $R^{N}$. Let $T=[0, b]$. A multifunction $F: T \times R^{N} \rightarrow P_{k}\left(R^{N}\right)$ is said to be graph measurable iff $\operatorname{GrF}=\left\{(t, x, v) \in T \times R^{N} \times R^{N}: v \in F(t, x)\right\} \in$ $\mathcal{L} \times B\left(R^{N}\right) \times B\left(R^{N}\right)$ with $\mathcal{L}$ being the Lebesgue $\sigma$-field of $T$ and $B\left(R^{N}\right)$ the Borel $\sigma$-field of $R^{N}$. A graph measurable multifunction $F(t, x)$ has the property that if $x: T \rightarrow R^{N}$ is measurable, then $t \rightarrow F(t, x(t))$ is graph measurable; i.e. $\operatorname{GrF}(\cdot, x(\cdot)) \in \mathcal{L} \times B\left(R^{N}\right)$. So by Aumann's selection theorem (see Wagner [12], Theorem 5.10), we can find a measurable function $g: T \rightarrow R^{N}$ such that $g(t) \in$ $F(t, x(t))$ a.e. on $T$.

Received by the editors September 23, 1996.

1991 Mathematics Subject Classification. Primary 34C25, 34A60.

Key words and phrases. Lower semicontinuous multifunction, measurable multifunction, continuous selector, a priori bound, compact embedding, Leray-Schauder degree, compact homotopy, homotopy invariance.

The second author's research was supported by Grant PENED 678(94).

(c)1999 American Mathematical Society 
Let $X, Y$ be Hausdorff topological spaces. A multifunction $G: X \rightarrow 2^{Y} \backslash\{\emptyset\}$ is said to be lower semicontinuous (abbreviated as l.s.c.), if for every open subset $U$ of $Y$, the set $G^{-}(U)=\{x \in X: G(x) \cap U \neq \emptyset\}$ is open in $X$. If $Y$ is a metric space, then this definition of lower semicontinuity is equivalent to saying that for all $y \in Y$, the $R_{+}$-valued function $x \rightarrow d(y, G(x))=\inf \left\{d_{Y}\left(y, y^{\prime}\right): y^{\prime} \in G(x)\right\}$ is upper semicontinuous (here $d_{Y}(\cdot, \cdot)$ denotes the metric on $Y$ ).

Finally, a subset $K$ of $L^{1}\left(T, R^{N}\right)$ is said to be decomposable, if for all $\left(A, g_{1}, g_{2}\right) \in$ $\mathcal{L} \times K \times K$, the function $\chi_{A} g_{1}+\chi_{T \backslash A} g_{2}$ belongs in $K$. Here by $\chi_{A}$ (resp. $\chi_{T \backslash A}$ ), we denote the characteristic function of $A$ (resp. of $T \backslash A$ ).

\section{MAin RESUlt}

In this section we establish the existence of an absolutely continuous function $x: T \rightarrow R^{N}$ which solves the following multivalued periodic problem:

$$
\left\{\begin{array}{l}
x^{\prime}(t) \in F(t, x(t)) \quad \text { a.e. on } T=[0, b], \\
x(0)=x(b) .
\end{array}\right.
$$

The hypotheses on $F(t, x)$ are the following:

$\mathbf{H}(\mathbf{F}) . F: T \times R^{N} \rightarrow P_{k}\left(R^{N}\right)$ is a multifunction such that

(i) $(t, x) \rightarrow F(t, x)$ is graph measurable;

(ii) $x \rightarrow F(t, x)$ is l.s.c. for every $t \in T$;

(iii) for every $r>0$ there exists $a_{r} \in L^{1}(T)$ such that for all $\|x\| \leq r$ we have $|F(t, x)|=\sup \{\|v\|: v \in F(t, x)\} \leq a_{r}(t)$ a.e. on $T$; and

(iv) there exists $\vartheta \in C^{1}\left(R^{N}, R\right)$ and $r>0$ such that $\vartheta^{\prime}(x) \neq 0$ for all $x \in R^{N}$ with $\|x\| \geq r, \quad-\left(\vartheta^{\prime}(x), x\right)_{R^{N}} \leq\left\|\vartheta^{\prime}(x)\right\|^{2}$ for all $x \in R^{N}$ (here by $(\cdot, \cdot)_{R^{N}}$ we denote the inner product in $\left.R^{N}\right), \vartheta(x) \rightarrow+\infty$ as $\|x\| \rightarrow+\infty$, if $x \in W^{1,1}\left(T, R^{N}\right)$ with $\min \{\|x(t)\|: t \in T\} \geq r$, then $\int_{0}^{b}\left(\vartheta^{\prime}(x(t)), g(t)\right)_{R^{N}} d t \leq 0$ for all $g \in$ $L^{1}\left(T, R^{N}\right), g(t) \in F(t, x(t))$ a.e. on $T$, and finally for almost all $t \in T$ and all $v \in F(t, x),\left(\vartheta^{\prime}(x), v\right)_{R^{N}} \leq \eta(t)$ with $\eta \in L^{1}(T)_{+}$.

Remark 3.1. If $\vartheta(\cdot)$ is convex and $\vartheta^{\prime}(0)=0$, then $\left(\vartheta^{\prime}(x), x\right)_{R^{N}} \geq 0$, so the hypothesis that $-\left(\vartheta^{\prime}(x), x\right)_{R^{N}} \leq\left\|\vartheta^{\prime}(x)\right\|^{2}$ is trivially true.

Theorem 3.1. If hypothesis $H(F)$ holds, then problem (1) has a solution.

Proof. Let $D=\left\{x \in W^{1,1}\left(T, R^{N}\right): x(0)=x(b)\right\}=W_{p}^{1,1}\left(T, R^{N}\right)$ and also let $L: D \subseteq L^{1}\left(T, R^{N}\right) \rightarrow L^{1}\left(T, R^{N}\right)$ be the linear operator defined by $L(x)=x^{\prime}+x$ for all $x \in D=W_{p}^{1,1}\left(T, R^{N}\right)$. In what follows by $\|\cdot\|$ we will denote the Euclidean norm of $R^{N}$ and by $\|\cdot\|_{*}$ the $l^{1}$-norm of $R^{N}$. Recall that the two are equivalent. So there exist $\vartheta_{1}, \vartheta_{2}, \vartheta_{3}, \vartheta_{4}>0$ such that $\vartheta_{1}\|\cdot\|_{*} \leq\|\cdot\| \leq \vartheta_{2}\|\cdot\|_{*}$ and $\vartheta_{3}\|\cdot\| \leq\|\cdot\|_{*} \leq \vartheta_{4}\|\cdot\|$. Then for $x(\cdot)=\left(x_{k}(\cdot)\right)_{k=1}^{N} \in D$ we have

$$
\begin{aligned}
\|L(x)\|_{1} & =\int_{0}^{b}\left\|x^{\prime}(t)+x(t)\right\| d t \geq \vartheta_{1} \int_{0}^{b}\left\|x^{\prime}(t)+x(t)\right\|_{*} d t \\
& =\vartheta_{1} \int_{0}^{b} \sum_{k=1}^{N}\left|x_{k}^{\prime}(t)+x_{k}(t)\right| d t=\vartheta_{1} \sum_{k=1}^{N} \int_{0}^{b}\left|x_{k}^{\prime}(t)+x_{k}(t)\right| d t .
\end{aligned}
$$


Fix $k \in\{1,2, \ldots, N\}$ and let $T_{k}^{+}=\left\{t \in T: x_{k}(t)>0\right\}$ and $T_{k}^{-}=\left\{t \in T: x_{k}(t)<\right.$ $0\}$. We have

$$
\begin{aligned}
\int_{0}^{b}\left|x_{k}^{\prime}(t)+x_{k}(t)\right| d t & \geq \int_{T_{k}^{+}}\left|x_{k}^{\prime}(t)+x_{k}(t)\right| d t+\int_{T_{k}^{-}}\left|x_{k}^{\prime}(t)+x_{k}(t)\right| d t \\
& \geq \int_{T_{k}^{+}}\left(x_{k}^{\prime}(t)+x_{k}(t)\right) d t-\int_{T_{k}^{-}}\left(x_{k}^{\prime}(t)+x_{k}(t)\right) d t \\
& =\int_{0}^{b}\left|x_{k}(t)\right| d t+\int_{T_{k}^{+}} x_{k}^{\prime}(t) d t-\int_{T_{k}^{-}} x_{k}^{\prime}(t) d t .
\end{aligned}
$$

Let $\left(a_{k}, d_{k}\right)$ be a component of $T_{k}^{+}$. Then since $x_{k}\left(d_{k}\right)=x_{k}\left(a_{k}\right)=0$, we have $\int_{a_{k}}^{d_{k}} x_{k}^{\prime}(t) d t=0$, from which it follows that $\int_{T_{k}^{+}} x_{k}^{\prime}(t) d t=0$ (note that if $[0, a) \subseteq T_{k}^{+}$, then $\left.(d, b] \subseteq T_{k}^{+}\right)$. Similarly we obtain that $\int_{T_{k}^{-}} x_{k}^{\prime}(t) d t=0$. Hence we deduce that

$$
\int_{0}^{b}\left|x_{k}(t)\right| d t \leq \int_{0}^{b}\left|x_{k}^{\prime}(t)+x_{k}(t)\right| d t .
$$

Using inequality (3) in (2), we have

$$
\begin{aligned}
\|L(x)\|_{1} & \geq \vartheta_{1} \sum_{k=1}^{N} \int_{0}^{b}\left|x_{k}(t)\right| d t=\vartheta_{1} \int_{0}^{b}\|x(t)\|_{*} d t \\
& \geq \vartheta_{1} \vartheta_{3} \int_{0}^{b}\|x(t)\| d t=\vartheta_{1} \vartheta_{3}\|x\|_{1} .
\end{aligned}
$$

For every $u \in L^{1}\left(T, R^{N}\right)$, the periodic problem $x^{\prime}(t)+x(t)=u(t)$ a.e. on $T, x(0)=$ $x(b)$, has a unique solution (see Mawhin [8], p. 72). So $L(\cdot)$ is surjective. Then from Theorem 2.11.6, p. 42, of Hille-Phillips [5], we have that $L^{-1}: L^{1}\left(T, R^{N}\right) \rightarrow$ $L^{1}\left(T, R^{N}\right)$ is a continuous linear operator. Evidently the range of $L^{-1}$ is $D=$ $W_{p}^{1,1}\left(T, R^{N}\right)$.

Claim. $L^{-1}: L^{1}\left(T, R^{N}\right) \rightarrow L^{1}\left(T, R^{N}\right)$ is completely continuous (i.e. it is continuous and maps bounded sets into relatively compact sets).

To this end let $K \subseteq L^{1}\left(T, R^{N}\right)$ be bounded. We will show that $L^{-1}(K)$ is relatively compact in $L^{1}\left(T, R^{N}\right)$. For this purpose let $x \in L^{-1}(K)$. Then $x=$ $L^{-1}(u)$ with $u \in K$, so $\|L(x)\|_{1}=\|u\|_{1}$. But we saw in the beginning of the proof that $\|x\|_{1} \leq\|L(x)\|_{1}$. Hence $\|x\|_{1} \leq|K|=\sup \left\{\|u\|_{1}: u \in K\right\}<+\infty$ and then $\left\|x^{\prime}\right\|_{1} \leq\|u\|_{1}+\|x\|_{1} \leq 2|K|$. From these bounds we infer that $L^{-1}(K)$ is bounded in $W_{p}^{1,1}\left(T, R^{N}\right)$. But $W_{p}^{1,1}\left(T, R^{N}\right)$ is embedded compactly in $L^{1}\left(T, R^{N}\right)$ (see for example Brezis [3]). Therefore $L^{-1}(K)$ is relatively compact in $L^{1}\left(T, R^{N}\right)$. Also if $u_{n} \rightarrow u$ in $L^{1}\left(T, R^{N}\right)$ as $n \rightarrow \infty$, then $\left\{L^{-1}\left(u_{n}\right)=x_{n}\right\}_{n \geq 1}$ is relatively compact in $L^{1}\left(T, R^{N}\right)$ and $x_{n} \rightarrow x=L^{-1}(u)$ in $L^{1}\left(T, R^{N}\right)$ as $n \rightarrow \infty\left(\right.$ since $L^{-1}(\cdot)$ is continuous from $L^{1}\left(T, R^{N}\right)$ into $\left.L^{1}\left(T, R^{N}\right)\right)$. Therefore $x_{n} \rightarrow x=L^{-1}(u)$ in $L^{1}\left(T, R^{N}\right)$ as $n \rightarrow \infty$, proving the desired complete continuity of $L^{-1}: L^{1}\left(T, R^{N}\right) \rightarrow$ $L^{1}\left(T, R^{N}\right)$.

Now for $0<\lambda<1$ we consider the following problem:

$$
\left\{\begin{array}{l}
x^{\prime}(t) \in F(t, x(t))-\lambda \vartheta^{\prime}(x(t)) \quad \text { a.e. on } T, \\
x(0)=x(b) .
\end{array}\right.
$$

Claim. The solutions of (4) are bounded in $C\left(T, R^{N}\right)$, uniformly in $0<\lambda<1$. 
Suppose not. Then we can find $x_{n} \in W^{1,1}\left(T, R^{N}\right),\left\|x_{n}\right\|_{\infty} \geq n$ and $0<\lambda_{n}<1$ such that

$$
\left\{\begin{array}{l}
x_{n}^{\prime}(t)=g_{n}(t)-\lambda_{n} \vartheta^{\prime}\left(x_{n}(t)\right) \quad \text { a.e. on } T \\
x_{n}(0)=x_{n}(b)
\end{array}\right.
$$

for some $g_{n} \in L^{1}\left(T, R^{N}\right), g_{n}(t) \in F\left(t, x_{n}(t)\right)$ a.e. on $T, n \geq 1$. Taking the inner product with $\vartheta^{\prime}\left(x_{n}(t)\right)$ we obtain:

$$
\left(x_{n}^{\prime}(t), \vartheta^{\prime}\left(x_{n}(t)\right)\right)_{R^{N}}=\left(g_{n}(t), \vartheta^{\prime}\left(x_{n}(t)\right)\right)_{R^{N}}-\lambda_{n}\left\|\vartheta^{\prime}\left(x_{n}(t)\right)\right\|^{2} .
$$

Hence using hypothesis $\mathrm{H}(\mathrm{F})(\mathrm{iv})$, we have

$$
\frac{d}{d t} \vartheta\left(x_{n}(t)\right) \leq \eta(t) \quad \text { a.e. on } T \text {. }
$$

We extend $x_{n}(\cdot)$ and $\eta(\cdot)$ by $b$-periodicity on all of $R$. Then for all $s \in R$ and all $t \in[s, s+b]$, we have

$$
\vartheta\left(x_{n}(t)\right) \leq \vartheta\left(x_{n}(s)\right)+\int_{s}^{t} \eta(\tau) d \tau
$$

which implies that

$$
\max _{t \in T} \vartheta\left(x_{n}(t)\right) \leq \min _{s \in T} \vartheta\left(x_{n}(s)\right)+\|\eta\|_{1}
$$

If $t_{n} \in T, n \geq 1$, is such that $\left\|x_{n}\left(t_{n}\right)\right\|=\left\|x_{n}\right\|_{C\left(T, R^{N}\right)}$, then

$$
\vartheta\left(x_{n}\left(t_{n}\right)\right) \leq \max _{t \in T} \vartheta\left(x_{n}(t)\right)
$$

so by hypothesis $\mathrm{H}(\mathrm{F})(\mathrm{iv})$ we have that $\max _{t \in T} \vartheta\left(x_{n}(t)\right) \rightarrow+\infty$ and so

$$
\min _{s \in T} \vartheta\left(x_{n}(s)\right) \rightarrow+\infty \quad \text { as } n \rightarrow \infty
$$

Now let $s_{n} \in T$ be such that $\left\|x_{n}\left(s_{n}\right)\right\|=\min _{s \in T}\left\|x_{n}(s)\right\|$. Then we have

$$
\min _{s \in T} \vartheta\left(x_{n}(s)\right) \leq \vartheta\left(x_{n}\left(s_{n}\right)\right) \text {. }
$$

Since $\min _{s \in T} \vartheta\left(x_{n}(s)\right) \rightarrow+\infty$ as $n \rightarrow \infty$, it follows that

$$
\left\|x_{n}\left(s_{n}\right)\right\|=\min _{s \in T}\left\|x_{n}(s)\right\| \rightarrow+\infty \quad \text { as } n \rightarrow \infty .
$$

So we can find $n_{0} \geq 1$ such that $\min _{s \in T}\left\|x_{n}(s)\right\| \geq r$ for all $n \geq n_{0}$. Since $\vartheta^{\prime}\left(x_{n}(t)\right) \neq 0$ for all $t \in T, 0<\lambda<1$, from hypothesis $\mathrm{H}(\mathrm{F})(\mathrm{iv})$ we get that

$$
\begin{aligned}
0 & =\vartheta\left(x_{n}(b)\right)-\vartheta\left(x_{n}(0)\right) \\
& =\int_{0}^{b}\left(g_{n}(t), \vartheta^{\prime}\left(x_{n}(t)\right)\right)_{R^{N}} d t-\lambda_{n} \int_{0}^{b}\left\|\vartheta^{\prime}\left(x_{n}(t)\right)\right\|^{2} d t<0,
\end{aligned}
$$

which is absurd. So the claim is true and there exists $\rho_{1}>0$ independent of $0<\lambda<1$, such that $\|x\|_{C\left(T, R^{N}\right)} \leq \rho_{1}$ for all solutions $x(\cdot)$ of (4).

Next let $\rho>b \rho_{1}$ and set $\bar{B}_{\rho}=\left\{x \in L^{1}\left(T, R^{N}\right):\|x\|_{L^{1}\left(T, R^{N}\right)} \leq \rho\right\}$ and consider the multifunction $R: \bar{B}_{\rho} \rightarrow 2^{L^{1}\left(T, R^{N}\right)}$ defined by $R(x)=\left\{u \in L^{1}\left(T, R^{N}\right): u(t) \in\right.$ $F(t, x(t))$ a.e. on $T\}$. From the discussion in section 2 and hypothesis $\mathrm{H}(\mathrm{F})(\mathrm{iii})$, we see that $R(\cdot)$ has nonempty, closed and decomposable values. Also Theorem 3.5 of Papageorgiou [10] tells us that $R(\cdot)$ is l.s.c. Thus we can apply Theorem 3 of Bressan-Colombo [2] to obtain a continuous map $r: \bar{B}_{\rho} \rightarrow L^{1}\left(T, R^{N}\right)$ such that $r(x) \in R(x)$ for all $x \in \bar{B}_{\rho}$. 
Now define the map $h:[0,1] \times \bar{B}_{\rho} \rightarrow L^{1}\left(T, R^{N}\right)$ by

$$
h(\lambda, x)=(1-\lambda) L^{-1}\left(r(x)-\lambda \vartheta^{\prime}(x)+x\right) .
$$

From the complete continuity of $L^{-1}: L^{1}\left(T, R^{N}\right) \rightarrow L^{1}\left(T, R^{N}\right)$ established in the first claim, we see that $h(\lambda, x)$ is a compact homotopy. Moreover since by hypothesis $\mathrm{H}(\mathrm{F})(\mathrm{iv}),-\left(\vartheta^{\prime}(x), x\right)_{R^{N}} \leq\left\|\vartheta^{\prime}(x)\right\|^{2}$ for all $x \in R^{N}$, the estimation conducted in the proof of the second claim is still valid, so there exists an a priori bound independent of $0<\lambda<1$ for the solutions $x \in C\left(T, R^{N}\right)$ of $x=h(\lambda, x)$. Therefore exploiting the fact that $\operatorname{deg}_{L S}\left(I, \bar{B}_{\rho}, 0\right)=1$ and since the Leray-Schauder degree is homotopy invariant, we conclude that $h(0, x)=x$ has a solution $x \in C\left(T, R^{N}\right)$. But for $\lambda=0$, we obtain $L(x)=r(x)+x$, so $x^{\prime}(t)=r(x)(t) \in F(t, x(t))$ a.e. on $T, x(0)=x(b)$.

Remark 3.2. Let $F: T \times R^{N} \rightarrow P_{k}\left(R^{N}\right)$ be a multifunction satisfying hypotheses $\mathrm{H}(\mathrm{F})(\mathrm{i})$, (ii) and

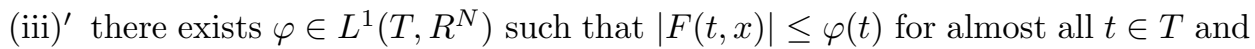
all $x \in R^{N}$.

Also let $K \in P_{k c}\left(R^{N}\right)=\left\{C \in P_{k}\left(R^{N}\right): C\right.$ is also convex $\}$ such that $\operatorname{int} K \neq \emptyset$. By translating things if necessary, we can always assume that $0 \in \operatorname{int} K$. Then if by $T_{K}(x)$ we denote the Bouligant tangent cone to $K$ at $x \in K$, we know that $\operatorname{int} T_{K}(x) \neq \emptyset$ and $x \rightarrow \operatorname{int} T_{K}(x)$ has open graph (see Aubin-Cellina [1], Proposition 4, p. 221). Then from Proposition 3.5 of Hu-Papageorgiou [6], we have that $\hat{F}(t, x)=\overline{F(t, x) \cap \operatorname{int} T_{K}(x)} \subseteq F(t, x) \cap T_{K}(x)$ (see Papageorgiou [9], Lemma $\gamma$ ) satisfies hypotheses $\mathrm{H}(\mathrm{F})(\mathrm{i})$, (ii) and

(iii) $|\hat{F}(t, x)| \leq \min \{\varphi(t),|K|\}$ where $|K|=\sup \{\|v\|: v \in K\}$.

Then if $\vartheta(x)=\frac{\|x\|^{2}}{2}$, we have that $\vartheta(\cdot)$ is convex and $\vartheta^{\prime}(x)=x$. So by virtue of Proposition 3, p. 220, of Aubin-Cellina [1], we see that hypothesis H(F)(iv) is satisfied with $r>0$ such that $\bar{B}_{r}(0) \subseteq K$. So according to our theorem here and since $\hat{F}(t, x) \subseteq F(t, x)$, the periodic problem $x^{\prime}(t) \in F(t, x(t))$ a.e. on $T$, $x(0)=x(b)$, has a solution. So we recover the result of Hu-Papageorgiou [6] when the "constraint" set $K$ is time independent. Note that in Hu-Papageorgiou [6], $K$ is time varying.

\section{REFERENCES}

1. J. P. Aubin and A. Cellina, Differential Inclusions, Springer-Verlag, Berlin, 1984. MR 85j: 49010

2. A. Bressan and G. Colombo, Extensions and selections of maps with decomposable values, Studia Math. 90 (1988), 69-86. MR 89j:54021

3. H. Brezis, Analyse Fonctionelle, Masson, Paris, 1983. MR 85a:46001

4. G. Haddad and J.-M. Lasry, Periodic solutions of functional-differential inclusions and fixed points of $\sigma$-selectionable correspondences, J. Math. Anal. Appl. 96 (1983), 295-312. MR 84m:34015

5. E. Hille and R. Phillips, Functional Analysis and Semigroups, AMS Colloq. Publ., Vol. 31, Amer. Math. Soc., Providence, RI, 1957. MR 54:11077

6. S. Hu and N. S. Papageorgiou, On the existence of periodic solutions for nonconvex valued differential inclusions in $R^{N}$, Proc. Amer. Math. Soc. 123 (1995), 3043-3050. MR 95m:34030

7. J. Macki, P. Nistri, and P. Zecca, The existence of periodic solutions to nonautonomous differential inclusions, Proc. Amer. Math. Soc. 104 (1988), 840-844. MR 89e:34027

8. J. Mawhin, Topological Degree Methods in Nonlinear Boundary Value Problems, CBMS, Regional Conference Series in Math., Vol. 40, Amer. Math. Soc., Providence, RI, 1979. MR 80c: 47055 
9. N. S. Papageorgiou, On infinite dimensional control systems with state and control constraints, Proc. Indian Acad. Sci. 100 (1990), 65-77. MR 91e:49018

10. N. S. Papageorgiou, On Fatou's lemma and parametric integrals for set valued functions, J. Math. Anal. Appl. 187 (1994), 809-825. MR 95m:28012

11. S. Plaskacz, Periodic solutions of differential inclusions on compact subsets of $R^{N}$, J. Math. Anal. Appl. 148 (1990), 202-212. MR 91d:34017

12. D. Wagner, Survey on measurable selection theorems, SIAM J. Control Optim. 15 (1977), 859-903. MR 58:6137

Department of Mathematics, Southwest Missouri State University, Springfield, MisSOURI 65804

E-mail address: hu@math.smsu.edu

Department of Mathematics, University of the Aegean, 83200 Karlovassi, Samos, Greece

Department of Mathematics, National Technical University, Zografou Campus, Athens 15780, GREece

E-mail address: npapg@math.ntua.gr 